\title{
SAÚDE BUCAL DE ADOLESCENTES ESCOLARES
}

\author{
A. C. G. FILGUEIRA', F. C. A. MACHADO'* , B. A. AMARAL ${ }^{2}$, K. C. LIMA e I. V. ASSUNÇÃO \\ ${ }^{1}$ Instituto Federal de Educação, Ciência e Tecnologia do Rio Grande do Norte \\ ${ }^{2}$ Universidade Federal do Rio Grande do Norte \\ flavitamachado@yahoo.com.br*
}

Artigo submetido em outubro/2015 e aceito em janeiro/2016

DOI: $10.15628 /$ holos.2016.3577

\begin{abstract}
RESUMO
Os problemas de saúde bucal podem impactar na qualidade de vida relacionada à saúde bucal. Assim, deve-se mensurar além da presença e severidade das doenças bucais, o quanto essas interferem no dia-a-dia das pessoas. Para tanto, utilizam-se indicadores de qualidade de vida relacionada à saúde bucal como Oral Impacts on Daily Performances. Na a dolescência, aumenta o risco de cárie e doença periodontal e de seus pos síveis impactos (dor de dente, dificuldades na mastigação e na higienização dentária), sendo in dicado o uso consorciado desses indicadores aos clínicos para o melhor planejamento das ações. Assim, realizou-se estudo seccional com 215 a dolescentes escolares (15-19 a nos) de um Instituto Federal de Educa ção, Ciência e Tecnologia para estimar sua condição de s a úde bucal e verificar a existência de impacto na qualidade de vida relacionada à saúde bucal. Pa ra tanto, fora m realizados exames clínicos e aplicados questionário para calcular, respectivamente, indicadores de cárie (índice cariado, perdido e obtura do- CPO-D), de periodontopatias (Índice Periodontal
\end{abstract}

Comunitário-CPI), odusopaticas (Índice de Estética Dental- DAl) e o Oral Impacts on Daily Performances. Como resultado, obteve-se um CPO-D de 3,31 $( \pm 4,15)$, o cál lculo dentário como periodontopatia mais severa e $31,5 \%$ da amostra com necessidade de tra tamento ortodôntico. Quan to à qualidade de vida relacionada à saúde bucal, $51,16 \%$ rela tou impacto cuja principal ca usa foi a posição dos dentes e relativo, sobretudo, às atividades de comer, higienizar a boca e sorrir. Os a dolescentes a presentaram em sua maioria dificuldades para realizar ao menos uma atividade diária relacionada à boca e a pres ença de cálculo dentário foi o agravo bucal mais prevalente. Es te dado associado às a tivi dades de execução mais dificultada (comer e realizar a higiene bucal) indica que há a necessidade de instituir, de fato, ações de promoção e prevenção à saúde. Portanto, a ções simples e de baixo custo como orientação de higiene bucal podem reduzir sensivelmente o impacto ocasionado no grupo pesquisado.

PALAVRAS-CHAVE: Qualidade de Vida, Saúde Bucal, Adolescente, Indicador de Saúde, Instituições de Ensino.

\section{ORAL HEALTH OF SCHOOL'S TEENAGER}

\begin{abstract}
Oral health problems can impact the Oral Health related Quality of Life. Thus, it's necessary to measure beyond the presence and severity of oral diseases, the interference of these diseases in the day-to-day people lives. To this end, Oral Health related Quality of Life indicators are used as the Oral Impacts on Daily Performances. In adolescence, increases the risk of caries and periodontal disease and its possible impacts (toothache, difficulty in chewing and dental hygiene), highlighting the importance of consortium use of these indicators to clinicians one for better action planning. Thus, there was conducted a cross-sectional study with 215 adolescent students (15-19 years) from a Federal Institute of Education, Science and Technology to estimate their oral health con dition and check its impact on Oral Health related Quality of Life. To this end, there were conducted clinical examination a nd ap plied questionnaire to calculate, res pectively, ca ries indicators (Decayed, Missed, Filled Tooth index - DMF-T), periodontal diseases (Community
\end{abstract}

Periodontal Index- CPI), malocclusion (Dental Aesthetic IndexDAI) and the Oral Impacts on Daily Performances. As a result, there was obtained a DMFT $3.31( \pm 4.15)$, the dental calculus was the more severe periodontal disease and $31.5 \%$ of the sample needed orthodontic treatment. As for Oral Health rela ted Quality of Life, $51.16 \%$ reported impact whose main cause was the position of the teeth and related mainly to the a ctivities of eating, clean the mouth and s mile. The ma jority of a dolescents presented difficulties in ca rrying out at least one daily activity related to the mouth and the presence of dental calculus was the most prevalent oral problem. This data associated with the activities with more execution difficulties rela ted (eating a nd performing oral hygiene) indicate that there is need to a ccomplish, in fact, promotion and prevention oral health actions. Therefore, simple and cost-effective actions as oral hygiene guidance can significantly reduce the impact caused in the group studied.

KEYWORDS: Quality of life, Oral Health, Adolescent, Health Status indicators, Schools. 


\section{INTRODUÇÃO}

A adolescência é um período crítico para a saúde, pois durante a transição da infância para a vida adulta, os indivíduos experimentam importantes mudanças biológicas, cognitivas, emocionais e sociais. Esta fase é um importante momento para adoção de novas práticas e comportamentos, ganho de autonomia, exposição a diversas situações e riscos presentes e futuros para a saúde geral e bucal (VETTORE et al., 2012).

Em relação à saúde bucal, há um aumento das doenças bucais biofilme dependentes (cárie e doença periodontal), particularmente nos subgrupos mais vulneráveis socioeconomicamente (VETTORE et al., 2012). Por sua vez, os problemas de saúde bucal têm sido cada vez mais reconhecidos como importantes causadores de impacto negativo no desempenho de atividades diárias e, consequentemente, na qualidade de vida dos indivíduos (GOMES; ABEGG, 2007).

Isto porque tais problemas podem acarretar dor de dente, dificuldades na mastigação, na higienização dentária, na pronúncia das palavras e insatisfação com a aparência facial (LEÃO; SHEIRAM, 1995; ANTUNES; LEÃO; MAIA, 2012). Assim, embora a maioria das doenças bucais não seja fatal, elas podem levar a uma significativa morbidade e impactos na qualidade de vida (COHEN-CARNEIRO; SOUZA-SANTOS; REBELO, 2011).

Desta forma, é importante mensurar não só a presença e severidade das doenças bucais através de indicadores clínicos, mas também mensurar os impactos que tais doenças acarretam no dia-a-dia das pessoas para realizarem suas atividades através de indicadores subjetivos. $O$ uso combinado de indicadores objetivos e subjetivos permitem um melhor dimensionamento da doença e suas consequências para os indivíduos, permitindo um planejamento mais adequado das intervenções (BIAZEVIC et al., 2008).

Os indicadores de qualidade de vida relacionados à saúde oral são definidos como os que mensuram o quanto problemas e desordens bucais interferem no funcionamento normal da vida das pessoas (LOCKER D; MILLER Y, 1994). Dentre esses indicadores, o Oral Impacts on Daily Performances (OIDP) já foi validado e utilizado no Brasil em diferentes estudos (GOMES; ABBEG, 2007; GOES, 2001; CORTES; MARCENES; SHEIHAM, 2002; MICHEL-CROSATO; BIAZEVIC; CROSATO,2005). O OIDP avalia a frequência e a severidade dos impactos odontológicos que afetam o desempenho das atividades diárias dos indivíduos, bem como os problemas bucais e sintomas causadores desse impacto (GOMES; ABBEG, 2007).

Por reconhecer os potenciais impactos na qualidade de vida que os agravos bucais podem ocasionar, a minimização ou prevenção dos mesmos se apresentam como importantes medidas de saúde pública. Uma vez que, na adolescência há potencialmente melhores índices de saúde, que podem ser mantidos e/ou melhorados frente à valorização e percepção que os adolescentes têm de saúde, o grupo dos adolescentes deve receber uma maior atenção das políticas públicas (GARBIN et al, 2009).

Neste sentido, a população jovem é um grupo prioritário para políticas de promoção da saúde em todas as regiões do mundo e a escola se constitui em um espaço privilegiado para implementação destas políticas (MOYSÉS et al., 2003). 
Este fato associado aos resultados do SB Brasil 2010 que revelam que 65,1\% dos adolescentes entre 15 e 19 anos auto-referiram necessidade de tratamento dentário, 24,7\% deste grupo relataram dor de dente nos 6 meses anteriores à pesquisa e que 13,6\% desses adolescentes nunca consultaram o dentista (BRASIL, 2011) demonstram a relevância de verificar a condição de saúde bucal de adolescentes estudantes de instituições que disponibilizam assistência odontológica gratuita a seus alunos como os Institutos Federais de Educação. Isto porque devemos averiguar se a assistência odontológica dessas instituições é relevante para melhorar a condição de saúde bucal dos brasileiros caracterizada por desigualdades regionais onde a dificuldade de acesso aos serviços é vista com um fator de risco.

E ainda, é preciso produzir informações que possam orientar o planejamento de intervenções e políticas de saúde nessas instituições em prol da maior efetividade dos serviços. Assim, será factível elaborar programas de ações coletivas em saúde bucal centrados na estratégia da definição dos grupos de risco, otimizando recursos e melhorando a resolutividades das intervenções (SANTOS; ALVES; FREITAS, 2007). Entretanto, enfatizamos que não basta produzir informações, é preciso difundir e institucionalizar o uso dessas informações para planejar e gerir os serviços de saúde como um todo.

Por tudo isso, o principal objetivo deste estudo foi investigar a condição de saúde bucal quanto à cárie dentária, doenças periodontais e má oclusão de adolescentes de 15 a 19 anos e dimensionar o impacto que essa condição acarreta no dia-a-dia deste grupo.

\section{MÉTODO}

Tal pesquisa se apresenta como um estudo de prevalência de desenho seccional realizado no Campus Central do Instituto Federal de Educação, Ciência e Tecnologia do município de Natal/RN. De modo geral, esses institutos têm como missão a educação superior, básica e profissional, tendo como característica a disponibilidade de vagas segundo princípios de equidade ( $50 \%$ das vagas são disponibilizadas para alunos da rede pública). E ainda, possuem uma política educacional que tem a qualidade de vida dos discentes como diretriz. Neste sentido, disponibilizam aos discentes atividades esportivas, religiosas e de estímulo à comunicação (grupos teatrais, corais de música) que potencializam a criação de uma rede apoio social. Além dessas atividades, detêm uma política de assistência estudantil que inclui assistência à saúde aos alunos regularmente matriculados (INSTITUTO FEDERAL DE EDUCAÇÃO, CIÊNCIA E TECNOLOGIA DO RIO GRANDE DO NORTE, 2012).

A população fonte deste estudo foi constituída pelo conjunto de 2.826 adolescentes de 15 a 19 anos regularmente matriculados no 2 o semestre de 2012 na instituição, correspondendo a $49,88 \%$ do total de alunos do IFRN. Do total de adolescentes, $51,84 \%$ são do sexo feminino (1465) e $48,16 \%$ do sexo masculino (1361).

A opção pela faixa etária de 15-19 anos foi feita com base nos critérios do Projeto SB Brasil 2010 para delimitar o grupo de estudo representativo do ciclo de vida da adolescência e assim, possibilitar a utilização dos dados deste levantamento como parâmetro. Entretanto, o principal motivo foi priorizar a segunda fase da adolescência posto que, a primeira fase (10-14 anos) é geralmente o foco das pesquisas e também dos programas governamentais como o Programa Saúde na Escola (BRASIL, 2009). 
Para realização do estudo, foi composta uma amostra de conveniência cujo tamanho foi estimado com base em resultado de estudo piloto realizado entre 15 e 27 de Junho de 2012 . 0 piloto apontou que $60 \%$ dos 30 alunos pesquisados relataram alguma dificuldade no desempenho de atividades diárias, em relação ao domínio bucal, coletadas por meio do questionário OIDP (ADULYANON; SHEIHAM, 1997). Sendo assim, a proporção de $60 \%$ do evento na população pesquisada, nível de confiança de $95 \%$, margem de erro de $12 \%$, acrescido de $20 \%$ para compensação de possíveis perdas amostrais resultou na definição de uma amostra de 214 sujeitos. No entanto, a amostra deste estudo foi de 215 adolescentes.

Para a obtenção dos dados relativos à condição de saúde bucal foram realizados exames clínicos no consultório odontológico do IFRN utilizando espelho bucal plano número cinco e sonda periodontal da OMS (CPI ou ball point) sobre condições adequadas de visibilidade (iluminação e campo seco). Tal exame visava estimar a experiência de cárie dentária em coroa através do Índice cariado, perdido e obturado para a unidade dente (CPO-D), de condição periodontal através do Índice Periodontal Comunitário (CPI) e de estética dentária através do Índice de Estética Dental (DAI). O indicador CPO-D é obtido através da contagem e registro do número de dentes cariados (C), perdidos devido à cárie $(\mathrm{P})$ e restaurados (O). Para o registro de prevalência da cárie dentária, a OMS preconiza o exame tátil-visual, onde um dente somente é considerado cariado quando uma lesão de fóssula, fissura ou superfície lisa tem uma cavidade evidente, esmalte socavado, ou um amolecimento detectável do assoalho ou das paredes dentárias. Neste estudo, somente o estado das coroas dentárias foram registradas (ANTUNES; PERES; FRAZÃO, 2006). Quanto ao CPI, a boca é dividida em sextantes e apenas um dente de cada sextante é examinado (dentes números 16, 11, 26, 36, 31 e 46) para verificar presença de sangramento, Cálculo dentário ou bolsa periodontal. Caso os dentes 16, 26, 36 e 46 estejam ausentes, o exame é realizada nos dentes números 17, 27, 37 e 47, respectivamente. O critério para avaliação do sextante é a presença de dois ou mais dentes sem indicação de exodontia (BASSANI; LUNARDELLI, 2006). Já o DAI busca detectar a presença de oclusopatias e o nível de necessidade de tratamento. Para isto, avalia presença de dentes ausentes (no es paço correspondente aos dentes números 15,14,13,12,11,21,22,23,24 e 25), apinhamento e espaçamento anterior, diastema na linha média, maior irregularidade anterior na maxila, maior irregularidade anterior na mandíbula (medidas em milímetros), trespasse horizontal maxilar mordida aberta anterior (medida em milímetro) e relação intermolar ântero-posterior (COSTA, 2007).

Enfatizemos que a coleta de dados foi precedida de treinamento e calibração do pesquisador responsável pela pesquisa que alcançou um Kappa intra-examinador $\geq 0,80$ para os índices CPI e DAl e de 1,0 para o CPOD. Tal processo ocorreu no intervalo de uma semana, com 10 adolescentes, segundo os critérios da OMS.

Para dimensionar o quanto a condição de saúde bucal interfere a realização de atividades diárias relacionadas ao domínio bucal dos adolescentes foi aplicado um questionário estruturado e autoaplicável baseado no indicador subjetivo OIDP.

O OIDP avalia a presença de dificuldades para: comer e apreciar a comida; falar e pronunciar claramente as palavras; realizar a higiene oral; dormir e relaxar; sorrir sem embaraço; manter o estado emocional usual sem irritabilidade; realizar o trabalho e/ou papel social; desfrutar do contato com as pessoas. Além da presença, são verificados a frequência, severidade, sintoma e causa relacionados à dificuldade (ADULYANON; SHEIHAM, 1997). 
Assim, o questionário OIDP consiste em cinco questões que versam sobre a presença de dificuldades para realizar as atividades mencionadas nos últimos 06 meses por problemas da boca, dente ou prótese (registrada como sim ou não), na presença de dificuldade, o indivíduo é questionado sobre a frequência e intensidade, seguido do questionamento sobre o sintoma e causa relacionados à dificuldade. A frequência e intensidade podem variar numa escala de zero a cinco, sendo zero a indicação de não saber responder e cinco a frequência e intensidade mais expressiva. Em relação ao sintoma, o indivíduo pode relatar não saber apontá-lo ou indicar dor, desconforto, limitação funcional e Insatisfação estética ou outros, especificando-os. Quanto às causas, havia opções no questionário relacionadas ao dente (ausência, cor, posição, forma, tamanho, mobilidade, dor), à restauração (cor, fratura), lesões na mucosa bucal (feridas bucais ou manchas), alterações fisiológicas (alteração de paladar, ardência na boca, barulhos na ATM, mandíbula travada), alterações anatômicas faciais (deformidade da boca ou rosto), alterações periodontais (abscesso gengival, recessão gengival, gengiva sangrenta), hálito desagradável, dispositivos protéticos (retenção deficiente) e ortodônticos. E ainda, era possível marcar a opção "não sabe" e "outros (especificar)".

Após a obtenção das respostas, o pesquisador faz o cálculo do impacto de cada uma das oito atividades, bem como o impacto total da condição da boca, dentes ou prótese na vida diária do indivíduo. O impacto de cada atividade é obtido pela multiplicação da frequência pela severidade indicada pelo indivíduo relacionada à dificuldade relatada. Já, o impacto total resulta da soma dos impactos de cada atividade, dividido pelo escore máximo que pode ser obtido nas oito atividades (200) e multiplicado por 100 (ADULYANON; SHEIHAM, 1997).

Além desses dados, foram coletados outros (Identificação se cursou o ensino fundamental em escola pública ou privada, renda familiar, escolaridade da mãe e moradia) para caracterização socioeconômica dos entrevistados a partir do banco de dados do IFRN.

Após a coleta dos dados foi feita a organização do banco de dados no programa PSPP e realizada análise descritiva dos mesmos.

Todo o protocolo deste estudo foi aprovado pelo Comitê de ética em Pesquisa da Universidade Federal do Rio Grande do Norte através do Parecer № 489/2011. Ressaltamos ainda, que os pais dos alunos participantes (se menores de 18 anos) ou os próprios alunos (se maiores de 18 anos) preencheram e assinaram um Termo de Consentimento Livre e Esclarecido.

\section{RESULTADOS}

Dos 215 adolescentes pesquisados, a maioria tinha entre 15 e 17 anos, com um pequeno predomínio do sexo feminino sobre o masculino, tendendo para uma homogeneidade entre os sexos. A maioria desses adolescentes mora com os pais, cursaram o ensino fundamental em uma instituição pública de ensino, possui mães que concluíram o ensino médio e suas famílias recebem entre dois e três salários mínimos de renda mensal bruta.

Quanto à condição de saúde bucal, foram registrados 61 pacientes $(28,4 \%)$ livres de cárie $(C P O D=0)$. Lesões de cárie não tratada foram observadas em 74 pacientes $(34,4 \%)$ e 65 adolescentes apresentaram dentes obturados (30,2\%). Apenas 15 adolescentes (7\%) apresentaram algum dente perdido em decorrência da cárie dentária. As médias e desvios padrões do CPO-D e seus componentes estão descritos na Tabela 1. 
Tabela 1- Médias e desvios padrões do CPO-D e seus componentes, dos adolescentes pesquisados. Natal/RN, 2013.

\begin{tabular}{c|c|c|c|c}
\hline & Cariados & Perdidos & Obturados & CPO-D \\
\hline Média & 0,93 & 0,10 & 2,31 & 3,31 \\
\hline Desvio padrão & 2,23 & 0,43 & 2,90 & 4,15 \\
\hline
\end{tabular}

Quanto à condição periodontal, enfatizemos que embora os seis dentes índices tenham sido examinados e registrados, apenas a pior condição foi considerada. A presença de cálculo dentário foi a condição periodontal mais prevalente entre os adolescentes examinados ( $n=124$, $57,7 \%)$, sendo o sextante central inferior o mais acometido. Apenas $0,5 \%(n=1)$ apresentou bolsa periodontal e $24,6 \%(n=53)$ apresentaram sangramento.

De acordo com o DAl, a maioria dos adolescentes examinados não possuíam necessidade real de tratamento ortodôntico ( $n=146 ; 68,5 \%)$.

Em relação à qualidade de vida relacionada à saúde bucal, pouco mais de $50 \%$ dos adolescentes relataram dificuldade para realizar ao menos uma atividade diária em decorrência de problemas odontológicos. A mediana do OIDP foi de 0,50 e seu valor total por indivíduo está distribuído entre os tercis: $Q_{25}=0,00, Q_{50}=0,50$ e $Q_{75}=5,00$.

Ainda em relação ao OIDP, este foi dicotomizado em duas categorias: ausência de impacto $(O I D P=0)$ e presença de impacto $(O I D P>0)$. Os resultados da análise descritiva do OIDP estão elencados na Tabela 2.

Tabela 2- Frequências absoluta e relativa do OIDP dos adolescentes pesquisados. Natal/RN, 2013.

\begin{tabular}{|c|c|c|c|c|c|c|c|c|}
\hline & \multicolumn{4}{|c|}{ Impacto } & \multicolumn{4}{|c|}{ Total } \\
\hline & \multicolumn{2}{|c|}{ Ausência } & \multicolumn{2}{|c|}{ Presença } & \multirow[b]{2}{*}{ Média } & \multirow[b]{2}{*}{$d p$} & \multirow[b]{2}{*}{ Mínimo } & \multirow[b]{2}{*}{ Máximo } \\
\hline & $n$ & $\%$ & $n$ & $\%$ & & & & \\
\hline $\begin{array}{l}\text { OIDP Total } \\
\text { por indivíduo }\end{array}$ & 105 & 48,84 & 110 & 51,16 & 3,25 & 5,47 & 0,00 & 38,00 \\
\hline
\end{tabular}

$\mathrm{dp}=$ Desvio padrão

Em relação às atividades cuja execução foi dificultada por problemas odontológicos, destacaram-se: comer, escovar os dentes ou higienizar a boca e sorrir (Gráfico 1).

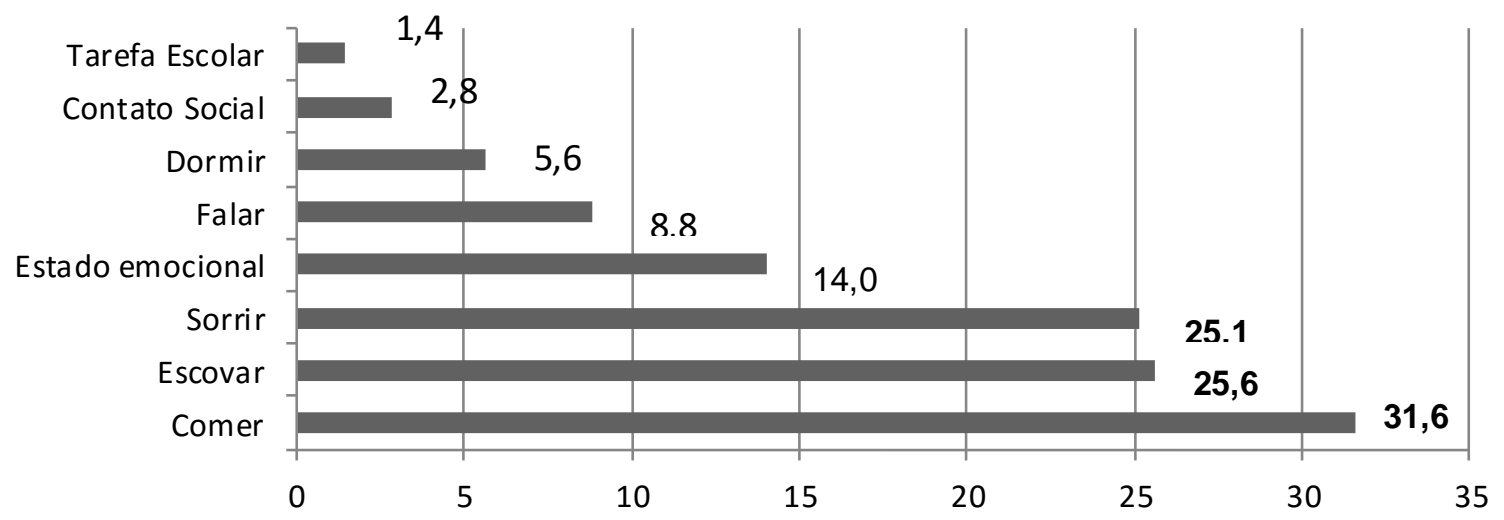

Gráfico 1- Frequência relativa do impacto na QVRSB por atividade diária, de acordo com o OIDP. Natal/RN, 2013.

Quanto à causa responsável pela dificuldade, houve preponderância de respostas indicando a posição dos dentes (Gráfico 2). 


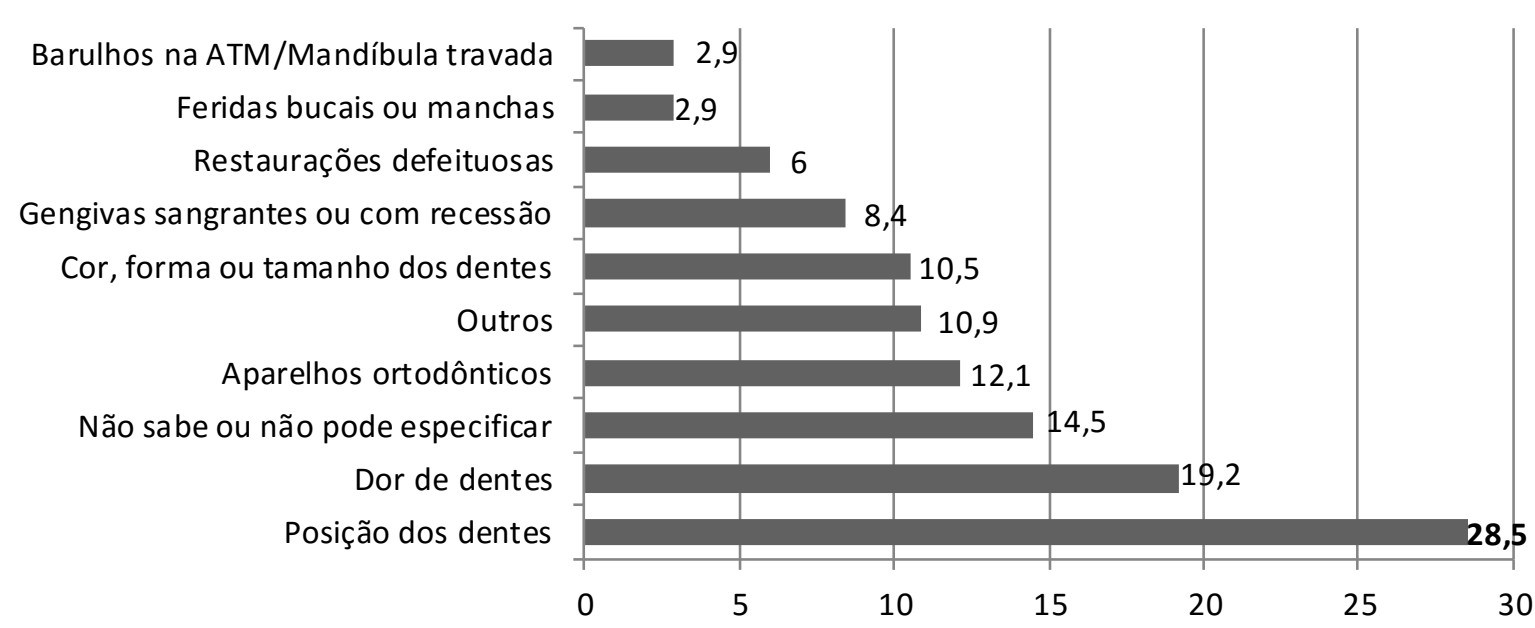

Gráfico 2 - Frequência relativa das causas odontológicas responsáveis pelo impacto na qualidade de vida dos adolescentes escolares. Natal/RN, 2013.

\section{DISCUSSÃO}

Na adolescência, os problemas bucais mais prevalentes ainda são os biofilme dependentes : cárie dentária, gengivite e cálculo dentário (BRASIL, 2011). Isto possivelmente se deve a maior independência em relação ao consumo de alimentos cariogênicos e cuidados inadequados quanto à higiene bucal. Por conseguinte, a adolescência pode representar um período de risco em relação à saúde bucal (GARBIN, 2009).

Por tudo isso, existe a necessidade de elucidar a proporção em que os problemas bucais intereferem no crescimento e desenvolvimento dos adolescentes, bem como a importância de estabelecer parâmetros aceitáveis de saúde bucal, numa perspectiva regional, respeitando características e peculiaridades locais (SANTOS et al., 2007). Por conseguinte, é preciso considerar a singularidade do indivíduo e também a da comunidade (SOUZA, 2006).

Quanto às necessidades e expectativas dos adolescentes, diversos estudos de autopercepção em saúde bucal demonstram que algumas condições clínicas são percebidas como situações que interferem em sua qualidade de vida. Em uma pesquisa com 332 adolescentes de 15 a 19 anos de idade, de ambos os sexos, matriculados em três escolas públicas, Bastos (2009) verificou que dor física e desconforto psicológico foram as dimensões que mais afetaram os adolescentes estudados. Além disso, foi evidenciado que tanto a prevalência como a morbidade dos agravos bucais mais comuns entre os adolescentes estão concentrados em populações mais carentes.

Em consonância com este resultado, o estudo vigente evidenciou que 51,16\% dos adolescentes de 15 a 19 anos relataram alguma dificuldade para realizar atividades. Portanto, evidenciaram impacto em sua qualidade de vida. E ainda, os impactos foram mais relatados nas atividades de comer, realizar a higiene bucal e sorrir, ou seja, na dimensão física. Se considerarmos o quanto a capacidade de sorrir, influencia na forma de nos relacionarmos socialmente e no bemestar, poderíamos dizer que houve congruência com os resultados de Bastos (2009).

De fato, esses resultados eram esperados uma vez que, cada vez mais a boca apresenta uma dimensão social ampliada na vida das pessoas, influenciando em sua forma de interagir com 
o outro e na sua auto-aceitação. Isto é especialmente relevante na fase da adolescência onde habitualmente se processam mudanças estéticas e fisiológicas, como o aparecimento de es pinhas, que podem inibir esta interação e aceitação. Assim, mudanças adicionais ou diferenças do adolescente em relação grupo social no qual convive podem acarretar impactos significativos em sua qualidade de vida.

Em relação à concentração das doenças bucais biofilme dependentes em populações mais carentes, o estudo em questão não permite tal constatação, uma vez que $76,8 \%(n=165)$ da amostra tinha renda de 1 a 3 salários mínimos, tendendo a uma homogeneidade. Contudo, a maior frequência entre populações mais vulneráveis economicamente é comprovada. Freire et al (2012) ao estimar a prevalência da dor de dente em adolescentes (idades entre 11 e 17 anos ou mais) das 27 capitais brasileiras e analisar fatores sociodemográficos e comportamentais associados, utilizando os dados da Pesquisa Nacional de Saúde do Escolar (PeNSE) de 2009 argumentaram que relação entre privação econômica e dor de dente pode ser resultado da maior exposição dos menos privilegiados aos fatores de risco para os problemas bucais, tais como a dieta cariogênica, as deficiências no estado imunitário e nutricional, e o baixo acesso aos fluoretos, recursos de higiene bucal e serviços de saúde.

Partindo-se para análise das frequências dos agravos e doenças bucais investigados neste estudo, percebe-se congruência entre estudos anteriores. Assim, em relação à cárie dentária, Gushi et al (2005) ao delinear o seu perfil epidemiológico em adolescentes de 15 a 19 anos de idade no Estado de São Paulo encontraram que o componente obturado constituiu $71,26 \%$ do índice CPO-D, ao passo que os componentes cariado e perdido constituíram, respectivamente, $22,24 \%$ e $6,5 \%$. Portanto, resultados próximos ao estudo em questão.

Quanto às doenças periodontais, os resultados deste estudo foram congruentes ao levantamento de base nacional SB Brasil 2010 onde a presença de cálculo dentário foi a alteração periodontal mais prevalente entre adolescentes de 15 a 19 anos de idade $(28,4 \%)$ e cerca de $1 / 3$ dos adolescentes examinados apresentaram sangramento gengival (BRASIL, 2011). Portanto, podemos inferir que as ações mais demandadas por este grupo são as de educação em saúde. Isto porque uma higienização oral adequada seria a ação indicada para evitar tanto o acúmulo de cálculo dentário como a inflamação da gengiva; causa do sangramento.

A educação em Saúde tem o objetivo de oferecer instrumentos para fortalecer a autonomia dos usuários no controle do processo saúde-doença e na condução de seus hábitos com vista à melhoria da sua qualidade de vida (TURRIONI et al., 2012). Neste sentido, o contexto escolar dos adolescentes deste estudo propiciaria potencialmente maiores chances de atuação na perspectiva da educação em saúde. Isto porque seria possível realizar atividades integradas entre os profissionais da assistência à saúde do Instituto e os docentes, caracterizando a intersetorialidade, a interdisciplinaridade e a relação horizontal. Assim, poderiam ser incluídas temáticas nas salas de aula relacionadas à saúde, mas contextualizadas ao conteúdo disciplinas tradicional. Neste sentido, poderia incluir a discussão sobre as doenças bucais infectocontagiosas como a cárie dentária nas aulas de biologia, discussão sobre a determinação social das doenças bucais e a influência do território nas aulas de geografia. Ainda seria possível desenvolver rodas de conversa, procurando trabalhar com o universo simbólico dos adolescentes, sensibilizando-os da melhor forma em relação aos temas sobre saúde-doença-cuidado. 
Segundo Elias et al (2001), os fatores que mais motivam os adolescentes para cuidar de sua saúde bucal são: a aparência pessoal, a sexualidade, o emprego e a saúde de um modo geral. Isto porque a importância da saúde bucal para os adolescentes perpassa aspectos físicos, psicológicos e sociais. No aspecto físico são percebidas questões como a presença da "dor", da necessidade de poder "mastigar em ordem", do perigo de "câncer bucal", por sua vez, na dimensão psicológica, destaca-se a importância da aparência pessoal, em "estar mais bonito para as meninas", em "não ser mais xingada de dentuça", em "ter os dentes bonitos para beijar". Na dimensão social, relatam constrangimento em "por causa do mau hálito tem que conversar meio afastado".

De modo geral, a estratégia para atuar com o público dos adolescentes nas ações de saúde é desenvolver canais para uma comunicação dialógica pautada na discussão e reflexão em oposição às práticas de comunicação unidirecional, dogmática e autoritária com foco na transmissão de informação. Neste sentido, é de suma importância o processo de capacitação e o planejamento conjunto das ações entre docentes, pedagogos e os profissionais de saúde (TURRIONI et al., 2012). Neste aspecto, os Institutos Federais de Educação, Ciência e Tecnologia são ambientes de grande potencial para desenvolver tais estratégias por contarem com equipes multiprofissionais que incluem assistencial social, psicólogo, pedagogo, odontólogo, médicos, enfermeiros e pessoal auxiliar de saúde bucal e enfermagem. Contudo, o desafio ainda é a interdisciplinaridade e, sobretudo, a intersetorialidade. Não obstante, Horta e Sena (2010) defendem que a parceria com as comunidades locais, com os equipamentos sociais aos quais os jovens se vinculam, com os programas oriundos de diferentes setores, atrelada a um compromisso político, pode resultar em práticas de impacto na configuração do quadro de morbi-mortalidade.

Frise-se que as políticas e programas de saúde pública, no geral, têm este desafio em comum. Quanto aquelas direcionadas ao público adolescente, ainda prepondera um delineamento com base no perfil de risco e vulnerabilidades, deixando de abordar fatores relacionados a sua identidade social, contexto de vida e os recursos de acesso dos jovens que poderiam minimizar essas vulnerabilidades.

Assim, a principal contribuição deste estudo é propiciar este debate acerca da necessidade de extrapolar ações eminentemente curativas voltas a processos agudos, posto que cada vez mais, a sociedade como um todo demanda uma atuação voltada ao perfil de tripla carga de doenças onde se observam doenças agudas, crônicas e os agravos à saúde relacionados a causas externas. As doenças crônicas e agravos, em processo contínuo de ascendência, demandam processos de trabalho balizados essencialmente no referencial da promoção de saúde e no desenvolvimento de ações intersetoriais.

\section{CONCLUSÃO}

Os adolescentes deste estudo apresentaram em sua maioria dificuldades para realizar ao menos uma atividade diária relacionada ao domínio bucal e a presença de cálculo dentá rio foi a doença ou agravo bucal mais prevalente. Este dado associado às atividades de execução mais dificultada pelas condições de saúde bucal (comer e realizar a higiene bucal) nos indica que há a necessidade de instituir, de fato, ações de promoção e prevenção à saúde. Além disso, se relacionarmos a causa mais relatada como responsável pelas dificuldades (posição dos dentes) e a baixa necessidade de tratamento de má oclusão dentária entre os adolescentes há um reforço a esta afirmação. 
Em contrapartida, há uma reafirmação sobre o cuidado inadequado com a higiene no grupo de adolescentes, uma vez que a forma mais efetiva de controle do biofilme dentário - agente etiológico das periodontites sob estudo - a higienização bucal adequada com uso de fio dentário, escova e dentifrício, possivelmente atenderiam às demandas assistenciais desses indivíduos, minimizando os relatos de dificuldade e, por conseguinte, melhorando a qualidade de vida relacionada à saúde bucal.

Assim, cabe aos serviços de saúde captar essa peculiaridade do universo adolescente e reforçar ações de educação em saúde. Portanto, percebe-se a necessidade de repensar os modelos assistenciais vigentes, sobretudo, em ambientes assistenciais não vinculados, diretamente, ao sistema público de saúde brasileiro, tais quais os Institutos Federais de Educação.

Os Institutos Federais de Educação, Ciência e Tecnologia configuram-se em potenciais atores na instituição de modelos de atenção à saúde pautados na vigilância à saúde. Isto porque, essas instituições já desenvolvem projetos e atividades voltadas à constituição de uma rede de apoio social aos estudantes. Exemplos disto são as Diretorias e Coordenações de assistência estudantil que tem como função social viabilizar o acesso, permanência e êxito dos es tudantes e para isso, disponibilizam programas de bolsas aqueles em situação de maior vulnerabilidade socioeconômica. Inclusive, o setor de saúde está inserido nessas coordenações, uma vez que é compreendido que condições biopsicológicas interferem no desempenho acadêmico. Além disso, os IF têm uma diversidade de práticas esportivas e culturais como grupos de dança, teatro e música para auxiliar na expansão dos estudantes enquanto indivíduos e na construção da sua identidade social.

Portanto, iniciativas que busquem compreender o modo como os adolescentes vivem, pensam e percebem a vida são importantes para construir e legitimar esta identidade e facilitar instituírem-se intervenções de saúde mais efetivas e promotoras de cidadania (QUIROGA; VITALLE, 2013). O desenvolvimento deste estudo proporcionou o redirecionamento dos fatores avaliados no exame odontológico periódico dos estudantes que ingressam no IFRN, sendo incluído o indicador OIDP e perguntas relativas aos hábitos de higiene bucal. Além disso, o IFRN encontra-se em processo de reestruturação de suas ações de saúde caminhando para institucionalização de uma política de saúde própria, que segue valores éticos e técnico-científicos característicos dos profissionais de saúde e da política pública de saúde brasileira, o SUS, mas que considera seu contexto característico e potencialidades na missão de viabilizar o cuidado a seu público-alvo.

\section{REFERÊNCIAS BIBLIOGRÁFICAS}

1. ADULYANON, S., SHEIHAM, A. Oral Impacts on Daily Performances. In: SLADE, G. Measuring oral health and quality of life. Chapel Hill: University of North Carolina, Dental Ecology, 1997.

2. ANTUNES, J.L.F., PERES, M.A., FRAZÃO, P. Cárie dentária. In: ANTUNES, J.L.F., PERES, M.A. (ed.). Epidemiologia da Saúde Bucal. Rio de Janeiro: Guanabara Koogan, 2006. p. 49-67.

3. ANTUNES, L.A.A., LEÃO, A.T., MAIA, L.C. Impacto do traumatismo dentário na qualidade de vida de crianças e adolescentes: revisão crítica e instrumentos de medida. Ciência \& Saúde Coletiva, São Paulo, v.17, n. 12, p.3417-3424, 2012.

4. BASTOS, R.S. Impacto das condições de saúde bucal em relação à qualidade de vida de adolescentes escolares de 15 a 19 anos, numa dicotomia socioeconômica, no município de 
Bauru, São Paulo, em 2009. 154 f., il. Tese (Doutorado em Odontologia) - Faculdade de Odontologia de Bauru - Universidade de São Paulo, Bauru, 2009.

5. BASSANI, D., LUNARDELLI, A.N. Condições periodontais. In: ANTUNES, J.L.F., PERES, M.A. (ed.). Epidemiologia da saúde bucal. Rio de Janeiro: Guanabara Koogan, 2006. p. 68-82.

6. BIAZEVIC, M.G.H., et al. Relationship between oral health and its impact on quality of life among adolescents. Brazilian Oral Research, v.22, n. 1, p. 36-42, 2008.

7. BRASIL. Ministério da Saúde. Secretaria de Atenção à Saúde. Departamento de Atenção Básica. Coordenação Geral de Saúde Bucal. Projeto SB Brasil 2010: Resultados Principais. Brasília: Ministério da Saúde, 2011.

8. BRASIL. Ministério da Saúde. Secretaria de Atenção à Saúde. Departamento de Atenção Básica. Saúde na escola. Brasília: Ministério da Saúde; 2009. 96 p. : il. - (Série B. Textos Básicos de Saúde) (Cadernos de Atenção Básica ; n. 24).

9. COHEN-CARNEIRO, F., SOUZA-SANTOS, R., REBELO, M.A.B. Quality of life related to oral health: contribution from social factors. Ciência \& Saúde Coletiva, São Paulo,v. 16 (Supl.1), p. 10071015, 2011.

10. CORTES, M.I., MARCENES, W., SHEIHAM, A. Impact of traumatic injuries to the permanente teeth on theoral health-related quality of life in 12-14-year-old children. Community Dentistry and Oral Epidemiology, v. 30, p. 193-8, 2002.

11. COSTA, R.N. Necessidade de tratamento ortodôntico: validação do DAl (índice de estética dental) e do ICON (índice de complexidade, resultado e necessidade) para a região de Belo Horizonte. 2007.139 f., il. Dissertação (Mestrado em Odontologia) - Universidade Federal de Minas Gerais - Faculdade de Odontologia.

12. ELIAS, M.S., et al. A importância da saúde bucal para adolescentes de diferentes estratos sociais do município de Ribeirão Preto. Revista latino-americana de enfermagem, v. 9, n. 1, p. 88-95, 2001.

13. FREIRE, M.C.M., et al. Dor dentária e fatores associados em adolescentes brasileiros: a Pesquisa Nacional de Saúde do Escolar (PeNSE), Brasil, 2009. Cadernos de Saúde Pública, v. 28, Suppl 0, p. S133-S145, 2012.

14. GARBIN, C.A.S., et al. A saúde na percepção do adolescente. Physis: Revista de Saúde Coletiva. v. 19, n. 1,p.227-238, 2009.

15. GOES, P.S.A. The prevalenceand impact of dental pain in Brazilian schoolchildren and their families [dissertation doctoral]. London: University College London, Department of Epidemiology and Public Health, 2001.

16. GOMES, A. S., ABEGG, C. O impacto odontológico no desempenho diário dos trabalhadores do departamento municipal de limpeza urbana de Porto Alegre, Rio Grande do Sul, Brasil. Cadernos de Saúde Pública, v. 23, n. 7, p.1707-1714, 2007.

17. GUSHI, L.L., et al. . Cárie dentária em adolescentes de 15 a 19 anos de idade no Estado de São Paulo, Brasil, 2002. Cadernos de Saúde Pública, v. 21, n. 5, p. 1383-1391, 2005.

18. HORTA, N.C., SENA, R.R. Abordagem ao adolescente e ao jovem nas políticas públicas de saúde no Brasil: um estudo de revisão. Physis, v. 20, n. 2, p. 475-495, 2010.

19. INSTITUTO FEDERAL DE EDUCAÇÃO, CIÊNCIA E TECNOLOGIA DO RIO GRANDE DO NORTE. 
Projeto político-pedagógico do IFRN: uma construção coletiva. Natal, 2012.

20. LEÃO, A., SHEIHAM, A. Relation between Clinical Dental Status and Subjective Impacts on Daily Living. Journal of Dental Research. V.74, n.7, p.1408-13, 1995.

21. LOCKER, D., MILLER, Y. Subjectively reported oral health status in an adult population. Community Dentistry and Oral Epidemiology, v.22, n.6, p.425-30, 1994.

22. MICHEL-CROSATO, E., BIAZEVIC, M.G., CROSATO, E. Relations hip between dental fluorosis and quality of life: a population based study. Brazilian Oral Reserach, v.19, n.2, p. 150-5, 2005.

23. MOYSÉS, S.T., et al. Associations between health promoting schools policies on some indicators of oral health. Health Promotion International, v. 18, p. 209-18, 2003.

24. QUIROGA, F.L., VITALLE, M.S.S. $O$ adolescente e suas representações sociais: apontamentos sobre a importância do contexto histórico. Physis, v. 23, n. 3, p. 863-878, 2013.

25. SANTOS, N.C.N., ALVES, T.D.B., FREITAS, V.S. A saúde bucal de adolescentes: aspectos de higiene, de cárie dentária e doença periodontal nas cidades de Recife, Pernambuco e Feira de Santana, Bahia. Ciência \& Saúde Coletiva, São Paulo, v. 12, n. 5, p. 1155-1166, 2007.

26. SOUZA, E.C.F. Bucalidade: conceito-ferramenta de religação entre clínica e saúde bucal coletiva. Ciência \& Saúde Coletiva, São Paulo, v.11, n.1, p. 18-43, 2006.

27. TURRIONI, A.P.S., et al. Avaliação das ações de educação na saúde bucal de adolescentes dentro da Estratégia de Saúde da Família. Ciência \& Saúde Coletiva, v. 17, n. 7, p. 1841-1848, 2012.

28. VETTORE, M.V., et al. Condição socioeconômica, frequência de escovação dentária e comportamentos em saúde em adolescentes brasileiros: uma análise a partir da Pesquisa Nacional de Saúde do Escolar (PeNSE). Cadernos de Saúde Pública, v. 28, p. S101-S113, 2012. 